\title{
Mechanism of DRAM1's Regulation of Autophagy and Apoptosis
}

Xing-Ding Zhang*

Department of Lymphoma/Myeloma, The University of Texas MD Anderson Cancer Center, Houston, TX, USA

*Corresponding author: Xing-Ding Zhang, Department of Lymphoma/Myeloma, The University of Texas MD Anderson Cancer Center, 7455 Fannin St., Unit 903 , Houston, TX 77054, USA, Tel: +1713 563-3406; E-mail: xzhang13@mdanderson.org

Received date: August 10, 2015; Accepted date: August 11, 2015; Published date: August 14, 2015

Copyright: $\odot 2015$ Zhang XD. This is an open-access article distributed under the terms of the Creative Commons Attribution License, which permits unrestricted use, distribution, and reproduction in any medium, provided the original author and source are credited.

\section{Editorial}

Recent studies have shown that p53, a well-known tumor suppressor gene, plays a direct role in the inhibition of autophagy [1-3]. Autophagy is a membrane-trafficking process that mediates the delivery of cytoplasmic constituents to the lysosome for degradation [4]. DNA-Damage Regulated Autophagy Modulator 1 (DRAM1), a novel TP53 target gene, is an evolutionarily conserved lysosomal protein that has been reported to play an essential role in TP53dependent autophagy activation and apoptosis [1]. However, the mechanisms by which DRAM1 promotes autophagy and apoptosis are not fully understood. In this editorial article, I will elucidate the network by which DRAM1 regulates autophagy and apoptosis.

\section{DRAM1 Regulates Autophagy Flux through Lysosomes}

p53 induces successful autophagy in a DRAM1-dependent manner, and GFP-LC3 was found to diffuse within the cytoplasm, with occasional puncta representing the basal level of autophagosomes within the cell in the absence of DRAM1 [1]. Upon DRAM1 induction, an increase in GFP-LC3 puncta was observed, indicating a clear role for DRAM1 and p53 in the regulation of autophagy [1]. The mechanisms through which DRAM1 causes the formation of autophagosomes are not clear. DRAM1 may send a feedback signal to proteins that are involved in the initiation of autophagy; alternatively, it may work in conjunction with other factors downstream of p53 [5].

In the present study, I demonstrated that rapamycin increased the abundance of autophagosomes and that knock-down of DRAM1 reduced the rate autophagosome clearance after rapamycin withdrawal. The results also support the involvement of DRAM1 in the degradation of autophagosomes [6]. However, Galavotti et al found that DRAM1 was not involved in starvation- and mTORmediated autophagy activation [7]. Therefore, the role of DRAM1 in autophagy activation induced by other stimuli needs to be studied further. Lysosomes are rich in hydrolytic enzymes and are responsible for the degradation of intracellular materials captured by autophagy [8]. The increase in acidic lysosomes was noticeable, as indicated by a fluorescent dye.

Knock-down of DRAM1 resulted in the impairment of lysosomal acidification and the accumulation of LC3-II, indicating reduced autophagy flux [6]. DRAM1 appeared to regulate autophagy in both the early and later stages. It also increased the formation of and clearance of autophagosomes [6]. The mechanism of DRAM1 is a lysosomal protein, and it may regulate the dynamics of lysosomal membranes to increase V-ATPase activity and facilitate membrane recycling for autophagosomal formation.

\section{DRAM1 Regulates Apoptosis Involving Protein Levels and Lysosomal Localization of BAX}

DRAM1 was first reported as a TP53 target gene that modulated autophagy [1]. Although DRAM1 cannot induce apoptosis by itself, it is a critical component of TP53-induced apoptosis [1,5]. Classic apoptosis is triggered by the translocation of the proapoptotic protein BAX from the cytosol to the mitochondria and is induced by the release of cytochrome $c$, which leads to the activation of procaspase- 9 and -3 [9]. Bax, which is transcriptionally induced by activated p53, has been reported to be partially localized in lysosomes and to activate a lysosome-dependent apoptosis pathway [10].

Recently, studies showed that DRAM1 increased the protein levels of BAX independently of transcription; the role of DRAM1 in BAX protein levels provides a new molecular link between DRAM1 and apoptotic signalling [11]. There was a protein interaction between DRAM1 and BAX, and this interaction prolonged the half-life of BAX. Furthermore, upregulated DRAM1 recruited BAX to lysosomes, leading to the release of lysosomal cathepsin B and cleavage of BID (BH3-interacting domain death agonist) and cell death, partially through the lysosome-cathepsin B-tBid pathway [11].

\section{Conclusion}

In conclusion, DRAM1 regulates autophagosome clearance through promoting lysosomal acidification and the activation of lysosomal enzymes; the fusion of autophagosomes with lysosomes is important for autophagic degradation. On the other hand, DRAM1 may influence apoptosis through the upregulation of BAX and BAX lysosomal translocation. Thus, we have revealed a novel mechanism of crosstalk between autophagy and apoptosis that is mediated by DRAM1.

\section{References:}

1. Crighton D, Wilkinson S, O'Prey J, Syed N, Smith P, et al. (2006) DRAM, a p53-induced modulator of autophagy, is critical for apoptosis. Cell 126: 121-134.

2. Zhang XD, Wang Y, Wang Y, Han R, Wu JC, et al. (2009) p53 mediates mitochondria dysfunction-triggered autophagy activation and cell death in rat striatum. Autophagy 5: 339-350.

3. Zhang XD, Qin ZH, Wang J (2010) The role of p53 in cell metabolism. Acta Pharmacol Sin 31: 1208-1212.

4. Xie Z, Klionsky D (2007) Autophagosome formation: Core machinery and adaptations. Nat Cell Biol 9: 1102-1109.

5. Crighton D, Wilkinson S, Ryan K (2007) DRAM links autophagy to p53 and programmed cell death. Autophagy 3: 72-74.

6. Zhang XD, Qi L, Wu JC, Qin ZH (2013) DRAM1 regulates autophagy flux through lysosomes. PLoS One 8: e63245. 
Citation: Zhang XD (2015) Mechanism of DRAM1's Regulation of Autophagy and Apoptosis. J Pharmacogenomics Pharmacoproteomics 6: e147. doi:10.4172/2153-0645.1000e147

Page 2 of 2

7. Galavotti S, Bartesaghi S, Faccenda D, Shaked-Rabi M, Sanzone S, et al (2013) The autophagy-associated factors DRAM1 and p62 regulate cell migration and invasion in glioblastoma stem cells. Oncogene 32: 669-712.

8. de Duve C (1983) Lysosomes revisited. Eur J Biochem 137: 391-397.

9. Reed JC (1997) Double identity for proteins of the Bcl-2 family. Nature 387: $773-776$.
10. Johansson AC, Appelqvist H, Nilsson C, Kagedal K, Roberg K, et al. (2010) Regulation of apoptosis-associated lysosomal membrane permeabilization. Apoptosis 15: 527-540.

11. Guan JJ, Zhang XD, Sun W, Qi L, Wu JC, et al. (2015) DRAM1 regulates apoptosis through increasing protein levels and lysosomal localization of BAX. Cell Death Dis 6: e1624. 